\title{
Análisis técnico-táctico en Taekwondo con coordenadas polares a través del software HOISAN
}

\author{
Technical-tactical analysis in Taekwondo with polar \\ coordinates through software HOISAN
}

\section{Análise técnico-tático no Taekwondo com coordenadas polares através de software HOISAN}

\author{
José Antonio López-López ${ }^{1 *}$, Cristina Menescardi², Isaac Estevann ${ }^{2,3}$, Coral Falcó ${ }^{4}$ y Antonio Hernández-Mendo
}

${ }^{1}$ Universidad de Málaga, ${ }^{2}$ Universidad Católica de Valencia "San Vicente Mártir", ${ }^{3}$ Universitat de València, ${ }^{4}$ University of Bergen

Resumen: Se lleva a cabo un análisis de coordenadas polares en Taekwondo de las acciones realizadas por los hombres finalistas y semifinalistas en los Juegos Olímpicos de Londres 2012. Para este análisis, se consideraron como categorías focales las acciones relativas a la efectividad de uno, dos y tres puntos, y como categorías condicionadas variables técnico-tácticas para establecer las diferentes relaciones entre las diversas categorías relacionadas con el comportamiento técnico-táctico de los deportistas y la consecución de punto/s. Los resultados mostraron una relación, de excitación en las perspectivas retrospectiva y prospectiva, entre las conductas CAN (Acción de contraataque), CIR (Pierna de golpeo dirigida hacia el oponente) e IZQ (Golpeo realizado con la pierna izquierda) con respecto a la conducta focal EF1, mientras que las conductas GIR (Acción realizada con un giro previo) y ABI (la pierna delantera de ambos oponentes es diferente) lo están con la EF2; sugiriéndose así el entrenamiento de las citadas acciones con vistas a la consecución de dicha puntuación. Este estudio ha permitido comparar las relaciones que se establecen en la utilización de estrategias de interacción en el deporte de taekwondo. El análisis se ha realizado utilizando el software de observación HOISAN que ha permitido solventar las dificultades planteadas anteriormente en la realización y uso de esta técnica..

Palabras clave: Interacción, coordenadas polares, software HOISAN, observación, deporte de combate.

Abstract: The aim of the current research was to provide a novel perspective on the understanding of the actions performed in Taekwondo, to do this, the actions performed by the men finalists and semifinalists in the Olympic Games in London 2012 were analyzed. Based on a taxonomic and methodologically validated system, analysis of polar coordinates was performed using the genuine analytical technique. This technique allows us to know the relationship between the focal category and various conditional categories, in addition to build different behavioral maps. To perform the mentioned analysis, were chosen as focal categories those actions relating to the effectiveness of one, two and three points while as conditional categories were chosen technical and tactical variables. This innovative technique of analysis was implemented by using the observation software HOISAN. The software allows to remove the difficulties previously raised when using this technique.

Keywords: Interaction, polar coordinates, software HOISAN, observation, combatsport.

Resumo: Com o objetivo de compreender as açóes realizadas no Taekwondo analisaram-se as açóes dos atletas masculinos finalistas e semifinalistas nos Jogos Olímpicos de Londres 2012, com recurso à análise de coordenadas polares. Para esta análise, consideraram-se como categorías focais as ações relativas à obtenção de um, dois e três pontos, e como categorías condicionadas as variáveis técnico-táticas, de modo a estabelecer as diferentes relaçôes entre as diversas categorías relacionadas com o comportamento técnico-tático dos desportistas e o alcançar de ponto(s). Os resultados mostraram uma relação excitatória nas perspetivas retrospetiva e prospetiva, entre as condutas CAN (Ação de contra-ataque), CIR (pontapé circular) e IZQ (pontapé realizado com a perna esquerda) no que respeita à conduta focal EF1, enquanto que as condutas GIR (Ação realizada com uma rotaçáo prévia) e ABI (a perna adiantada dos dois adversarios é diferente) mostraram a mesma relação excitatória no que respeita à conduta EF2; sugere-se assim o treino das citadas açôes com vista à obtenção da pontuaçáo mencionada. Este estudo permitiu comparar as relaçóes que se estabelecem na utilização de estratégias de interação no Taekwondo. A análise realizou-se com recurso ao software de observação HOISAN que permitiu superar as dificuldades colocadas anteriormente na realizaçẫo e uso desta técnica.

Palavras-chave: Interação, coordenadas polares, software HOISAN, observação, desportos de combate.

\section{Introducción}

El taekwondo es un deporte olímpico desde el año 2000, practicado actualmente a nivel mundial por más de $30 \mathrm{mi}$ llones de personas en 188 países (Hermann, Scholz, Vieten y

Dirección para correspondencia [Correspodence address]: José Antonio López-López. Departamento de Psicología Social, A.S., T.S. y S.S., Facultad de Psicología, Campus de Teatinos. 29071. Málaga Universidad de Málaga, Málaga (España) E-mail: joseall93@gmail.com
Kohloeffel, 2008). Se trata de un deporte de combate, donde para conseguir puntuar se pueden utilizar técnicas de piernas o de brazo (WTF, 2012). Sin embargo, parece que las técnicas de pierna son más utilizadas durante la competición (Kazemi, Casella y Perri, 2009; Kazemi, Waalen, Morgan y White, 2006) debido a que hay más zonas donde impactar y permiten llegar más lejos, transfiriendo un mayor impacto al 
oponente, y aumentando así las posibilidades de conseguir puntuar (Kim, Kwon, Yenuga y Kwon, 2010). Los puntos se pueden conseguir por medio de acciones al tronco que proporcionan un punto, o acciones a la cabeza que proporcionan tres puntos. Además, si la acción técnica se realiza con un giro previo, se ańade un punto adicional (WTF, 2012). Puesto que el objetivo del taekwondo es conseguir la victoria en el combate por puntos o KO (WTF, 2012), cobra especial relevancia entender todo el entramado relacional que envuelve a la consecución de puntos.

Las acciones de pierna pueden llevarse a cabo durante una acción de ataque o de contraataque. Se considera que el ataque es iniciado por el deportista, mientras que el contraataque se produce cuando el oponente inicia una acción tras el ataque de su adversario. El ataque puede ser indirecto o directo en función de si le precede o no un recurso técnico. El contraataque puede ser anticipado, simultáneo o posterior al ataque del adversario (González, Iglesias, Mirallas y Esparza, 2011). Las acciones de ataque y/o contraataque han sido definidas, en función de su trayectoria, como técnicas circulares, lineales o con giro previo (Serina y Lieu, 1991; Falcó, Estevan, Álvarez, Morales-Sánchez y Hernández-Mendo, 2014). Las técnicas circulares son aquellas que van dirigidas a un lado del oponente mientras que, las técnicas lineales son aquellas que van dirigidas a la parte anterior del oponente. Finalmente, las técnicas con giro previo van precedidas de un giro mínimo de $180^{\circ}$ (Serina y Lieu, 1991). Otra característica diferencial de las técnicas de pierna, referida al golpeo, es la lateralidad -derecha e izquierda- (González, 2011), así como la pierna utilizada -adelantada o atrasada- para realizar la acción y, en función de la guardia -abierta o cerrada- en la que se encuentre el competidor (González et al., 2011).

En los últimos años han aumentado los estudios sobre el comportamiento técnico-táctico de los deportistas, con vistas a catalogar las conductas que estos realizan durante la competición (Casolino et al., 2012; Falcó, Landeo, Menescardi, Bermejo y Estevan, 2012; Falcó et al., 2014; Iglesias, Gasset, González y Anguera, 2010; Kazemi et al., 2006, Kazemi et al., 2009; Kwon, 2012; Matsushigue et al., 2009; Menescardi et al., 2012; Menescardi, López-López, Falcó, HernándezMendo y Estevan, 2015; Santos, Franchini y Lima-Silva, 2011; Tornello et al., 2013). La revisión de la literatura actual sugiere que los estudios en taekwondo únicamente analizan el parámetro frecuencia de dichas acciones. Apenas hay estudios donde se considere el parámetro orden o el tiempo de duración de dichas acciones (Camerino et al., 2014 y en este mismo monográfico consultar el trabajo de Iglesias, González y Anguera sobre detección de regularidades en taekwondo de alto nivel). Según Anguera, Blanco, Hernández-Mendo y Losada (2011), los datos que se pueden extraer de las conductas humanas, entre las que se incluye el comportamiento técnico-táctico de los taekwondistas, se basan en los parámetros frecuencia de acciones, orden de dichas acciones y duración de las mismas.

El análisis de coordenadas polares junto con el secuencial (i.e. técnica de retardos y markoviano), son característicos de la Metodología Observacional (Anguera y Hernández-Mendo, 2013, 2014), y utilizan el parámetro orden en su ejecución. Este tipo de técnica ha cobrado cada vez mayor relevancia dentro del mundo del deporte, llevándose a cabo en diversas disciplinas como fútbol, tenis o pelota vasca (Castellano y Hernández-Mendo, 2003; Castellano, Hernández-Mendo, Morales-Sánchez y Anguera, 2007; Gorospe, 1999; Hernández-Mendo y Anguera, 1998; Perea, 2008; Perea, Castellano, Alday y Hernández-Mendo, 2012; Usabiaga, 2005). El análisis de coordenadas polares tiene su origen en el trabajo de Sackett (1980) y posterior optimización con la "técnica genuina” (Anguera, 1997), que permite efectuar una reducción drástica de datos, así como una representación vectorial de las diferentes interrelaciones entre las distintas categorías que constituyen el sistema taxonómico elegido (Gorospe y Anguera, 2000; Hernández-Mendo y Anguera, 1999), que para el presente estudio se producen en los combates de Taekwondo pertenecientes a los Juegos Olímpicos.

Esta técnica se apoya en un análisis secuencial de retardos prospectivo (Sackett, 1980) y retrospectivo, con la técnica genuina (Anguera, 1997) de las sucesivas conductas ocurridas. Los valores obtenidos en el cálculo de la probabilidad condicionada permitirán la obtención del parámetro Zsum (Zsum $\left.=\Sigma_{\mathrm{z}} / \sqrt{\mathrm{n}}\right)($ Cochran, 1954), que actúa como potente reductor de datos siempre que se utilicen valores independientes como punto de partida (y aquí lo son, ya que cada uno de los valores de la probabilidad condicionada se ha hallado en paralelo). Para la obtención de los valores $Z$ sum se consideran retardos prospectivos (positivos) y retrospectivos (negativos). A partir de dichos valores se puede construir el mapa interrelacional de conductas, o mapa de coordenadas polares (Gorospe y Anguera, 2000), expresado mediante valores de módulo o longitud y ángulo, y representado en forma de vectores.

Considerando todo esto, el objetivo del presente estudio es establecer las diferentes relaciones entre las diversas categorías relacionadas con el comportamiento técnico-táctico de los taekwondistas y la consecución de punto/s en combate. Para conseguir el objetivo deseado, se realizó un análisis de coordenadas polares, se escogió como conductas focales aquellas relativas a la efectividad y como conductas condicionadas aquellas relativas a las acciones técnico-tácticas.

\section{Método}

Siguiendo la línea de trabajos previos, en análisis de coordenadas polares (Castellano y Hernández-Mendo, 2003; Castellano, Hernández-Mendo, Morales-Sánchez y Anguera, 2007; Hernández-Mendo y Anguera, 1998; Gorospe, 1999; 
Perea, 2008; Perea et al., 2012; Usabiaga, 2005) se ha utilizado un Diseño Observacional situado en el cuadrante IV de carácter nomotético, de seguimiento y multidimensional, (Anguera, Blanco, Hernández-Mendo y Losada, 2011).

\section{Participantes}

Se codificaron y registraron seis combates de los Juegos Olímpicos de Londres 2012. La elección del número de combates se estimó a través de un análisis de generalizabilidad (Blanco-Villaseñor, Castellano y Hernández-Mendo, 2000; Blanco-Villaseñor et al., 2014). Según el informe Belmont (1978) y siguiendo las normas establecidas por el Comité Ético, al ser vídeos públicos extraídos de medios de comunicación y no implicar experimentación de ningún tipo, no fue necesario el consentimiento informado por parte de los competidores.

\section{Instrumentos}

Instrumentos informáticos. Para realizar la codificación del flujo comportamental de los taekwondistas, se utilizó el programa informático HOISAN (Hernández-Mendo, López-López, Castellano, Morales-Sánchez y Pastrana, 2012; Hernández-Mendo et al., 2014). El programa permite tanto la codificación, como el análisis de coordenadas polares y su representación vectorial (Figura 1).

Figura 1. Formulario para el Cálculo de Coordenadas Polares del software HOISAN (Hernández-Mendo et al., 2012; HernándezMendo et al., 2014).

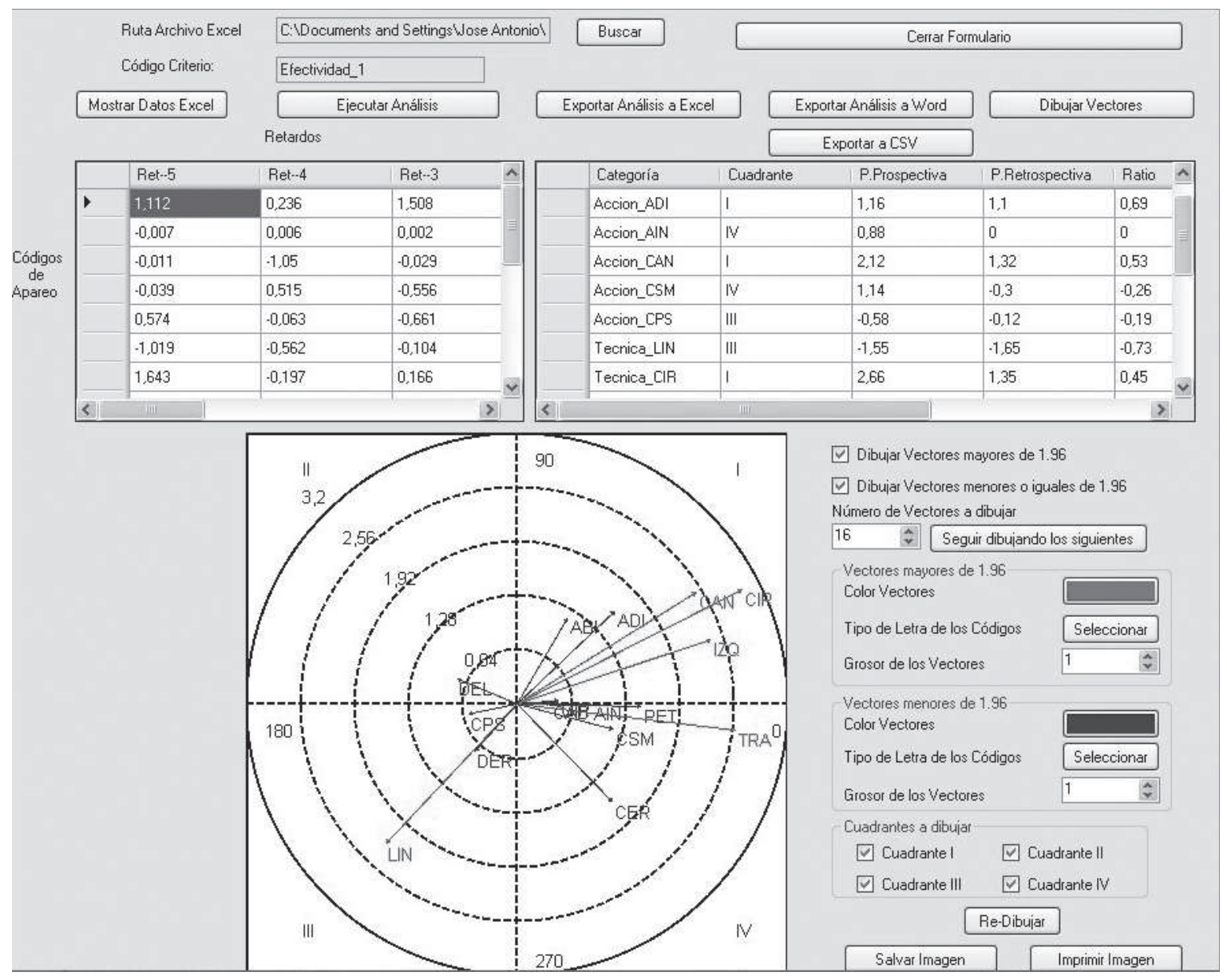

Instrumento de observación. En este trabajo se parte de un sistema mixto de formatos de campo y un sistema de categorías utilizado en trabajos previos (Falcó et al., 2012; Falcó et al., 2014; Menescardi et al., 2012; Menescardi et al., 2015). Las categorías focales utilizadas en el análisis de coordenadas polares pertenecen a la categoría de efectividad de las acciones, donde se considera la consecución de 1 punto (EF1), 2 puntos (EF2) y 3 puntos (EF3). No se ha dado en ningún caso la 
consecución de 4 puntos (acción con giro previo a la cabeza) durante la realización de los combates, por lo tanto dicha conducta (EF4) no será tenida en cuenta en el presente trabajo. Siguiendo la terminología específica de trabajos previos
(Falcó et al., 2012; 2014; González et al., 2011; Menescardi et al., 2012; Serina y Lieu, 1991), en la tabla 1 se presenta una breve definición de la conducta focal y de cada una de las conductas condicionadas.

Tabla 1. Sistema de observación empleado para el análisis de coordenadas polares.

\begin{tabular}{|c|c|c|}
\hline CRITERIO & CATEGORIAS & NÚCLEO CATEGORIAL \\
\hline \multirow[t]{3}{*}{ Efectividad } & EF1 & $\begin{array}{l}\text { Puntuación obtenida por una acción válida (con la suficiente fuerza y en las zonas permitidas de } \\
\text { golpeo) realizada al tronco con técnica lineal o circular. }\end{array}$ \\
\hline & EF2 & $\begin{array}{l}\text { Puntuación obtenida por una acción válida (con la suficiente fuerza y en las zonas permitidas de } \\
\text { golpeo) realizada al tronco con técnica de giro previo. }\end{array}$ \\
\hline & EF3 & $\begin{array}{l}\text { Puntuación obtenida por una acción válida (con la suficiente fuerza y en las zonas permitidas de } \\
\text { golpeo) realizada a la cabeza con técnica lineal o circular. }\end{array}$ \\
\hline \multirow[t]{5}{*}{ Acción táctica } & ADI & Acción de ataque iniciada con el objetivo de puntuar, el cual se realiza sin movimiento previo. \\
\hline & AIN & $\begin{array}{l}\text { Acción de ataque iniciada con un movimiento previo (i.e., salto, desplazamiento, cambio de pierna, } \\
\text { modificación de la trayectoria o zona de golpeo, etc.) con el objetivo de puntuar. }\end{array}$ \\
\hline & CAN & $\begin{array}{l}\text { Acción de contraataque iniciada durante el ataque del taekwondista y que finaliza en la fase } \\
\text { preparatoria (guardia) y/o fase inicial (fase de arranque) del oponente. Es decir, el oponente inicia } \\
\text { una acción de ataque, pero el taekwondista que contraataca acaba golpeando primero, por haberse } \\
\text { anticipado al ataque. }\end{array}$ \\
\hline & CSM & $\begin{array}{l}\text { Acción de contraataque iniciada al mismo tiempo o tras el ataque del oponente e impacta al mismo } \\
\text { tiempo que el ataque del oponente (fase de golpeo o impacto). }\end{array}$ \\
\hline & CPS & $\begin{array}{l}\text { Acción iniciada tras el ataque del oponente y que golpea cuando el oponente ya está descendiendo su } \\
\text { pierna (fase de caída) o después del apoyo de éste. A veces, incluye un desplazamiento previo hacía } \\
\text { atrás (esquiva). }\end{array}$ \\
\hline \multirow[t]{3}{*}{ Tipo de técnica } & LIN & $\begin{array}{l}\text { La pierna se dirige hacia la parte delantera del cuerpo del oponente, con un movimiento de empuje } \\
\text { y tratando de golpear con la planta del pie. }\end{array}$ \\
\hline & CIR & $\begin{array}{l}\text { La pierna de golpeo se dirige hacia el lado del oponente, con un movimiento oscilatorio y tratando } \\
\text { de golpear con el empeine del pie. }\end{array}$ \\
\hline & GIR & $\begin{array}{l}\text { Acción realizada con un giro previo, como mínimo de } 180^{\circ} \text { desde la posición inicial, antes golpear } \\
\text { al contrario. }\end{array}$ \\
\hline \multirow[t]{2}{*}{ Zona de golpeo } & $\mathrm{CAB}$ & Golpeo en las zonas permitidas de la cabeza. \\
\hline & PET & Golpeo en las zonas permitidas del tronco. \\
\hline \multirow[t]{2}{*}{ Lateralidad } & DER & Golpeo realizado con la pierna derecha. \\
\hline & IZQ & Golpeo realizado con la pierna izquierda. \\
\hline \multirow{2}{*}{$\begin{array}{l}\text { Pierna de } \\
\text { golpeo }\end{array}$} & DEL & Pierna que está más cercana al oponente. \\
\hline & TRA & Pierna que está más alejada al oponente. \\
\hline \multirow[t]{2}{*}{ Guardia } & ABI & $\begin{array}{l}\text { La pierna delantera de ambos oponentes es diferente (i.e., uno de ellos tiene adelantada la pierna } \\
\text { izquierda y el otro tiene adelantada la pierna derecha). }\end{array}$ \\
\hline & CER & $\begin{array}{l}\text { La pierna delantera de ambos oponentes es la misma (i.e., ambos oponentes tienen adelantada la } \\
\text { pierna izquierda). }\end{array}$ \\
\hline
\end{tabular}

\section{Procedimiento}

Previo a la codificación definitiva de los combates, ver ejemplo de tres registros en Figura 2, se realizó un análisis de la calidad del dato, donde se codificaron tres combates por tres observadores diferentes, todos ellos previamente entrenados.
En todos los casos, dentro del equipo, se utilizó la concordancia consensuada (Anguera, 1990). Una vez finalizada la codificación, se calculó la concordancia intra e interobservadores utilizando para ello el índice Kappa de Cohen (Cohen, 1960) y la concordancia canónica de Krippendorff (Krippendorff, 1980), obteniéndose en todos los casos valores superiores a 
0,75, asumiendo que el sistema de observación produce registros fiables (Banerjee, Capozzoli, McSweeney y Sinha, 1999;

Fleiss, 1981; Krippendorff, 2004; Landis y Koch, 1977).

Figura 2. Codificación de tres registros. Primera y segunda columna corresponden a Frame Inicial y Frame Final respectivamente, 12 columnas siguientes a cada uno de los criterios y la última columna al participante que efectúa el registro.

\begin{tabular}{|l|l|l|l|l|l|l|l|l|l|l|}
\hline Frame Inicial & Frame Final & Sexo & Peso & Evento & Asalto & Peto & Accion \\
\hline 2191 & 2205 & HOM & GAL & FIN & TAS & ROJ & ESQ \\
\hline 2332 & 2345 & HOM & GAL & FIN & TAS & ROJ & ADI \\
\hline 2345 & 2357 & HOM & GAL & FIN & TAS & AZU & CPS \\
\hline
\end{tabular}

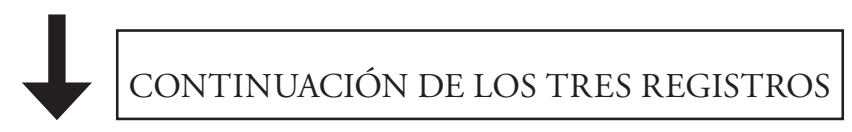

\begin{tabular}{|l|l|l|l|l|l|}
\hline Zona & Lateralidad & Piernagolpeo & Guardia & Efectividad & PARTICIPANTE \\
\hline SINC & SINC & SINC & SINC & SINC & 82 \\
\hline PET & DER & DEL & CER & 0 & 82 \\
\hline PET & DER & DEL & CER & 0 & 96 \\
\hline
\end{tabular}

La técnica de coordenadas polares (Sackett, 1980) parte de los valores Zsum en el número de retardos considerados (en este caso, -5 y 5) tanto para la perspectiva prospectiva como retrospectiva. Esta consideración permitirá establecer la existencia de dependencia excitatoria o inhibitoria (según sean los valores positivos o negativos), previa y posteriormente, entre la conducta focal y las conductas condicionadas (Santoyo y Anguera, 1993; Anguera, Espinosa y Santoyo, 2002; Anguera y Losada, 1999; Anguera, Santoyo y Espinosa, 2003; Espinosa, Anguera y Santoyo, 2004; Herrero, 2000). Estas últimas equivalen a una imagen especular de la última, penúltima, etc. conductas anteriores a la conducta criterio, o primera, segunda, tercera, etc. posteriores a la conducta criterio, dándose entre todas ellas una relación estable y, desvelándose como «preparatorias» o «resultantes» a la ocurrencia de la conducta criterio (Gorospe y Anguera, 2000).

A continuación se presenta, a modo de resumen, los cálculos realizados con la herramienta HOISAN (HernándezMendo et al., 2012; Hernández-Mendo et al., 2014):

1. Análisis secuencial usando la categoría focal y las distintas categorías condicionadas, obteniendo los Residuos Ajustados (Z) del rango de retardos $(-5,5)$. Estos Residuos Ajustados $(Z)$ se introducen en el formulario de "Análisis de Coordenadas Polares" (Figura 1). Los valores obtenidos han sido validados mediante el algoritmo de Matlab (Perea et al., 2012) y, siguiendo la línea de trabajos previos (Castellano y Hernández-Mendo, 2003), han sido hallados usando la técnica analítica de Sackett (1980) en su variante de retrospectividad genuina propuesta por Anguera (1997). Para ello, se han empleado las siguientes fórmulas:

I. Zsum prospectivo $(X)=$ Sumatorio de los residuos ajustados del retardo (1 al 5).

II. Zsum retrospectivo $(Y)=$ Sumatorio de los residuos ajustados del retardo $(-5$ al -1$)$.

III. Cuadrante del vector = Cuadrante en que se situará la categoría condicionada en función del valor positivo o negativo del Zsum de $\mathrm{X}$ y de $\mathrm{Y}$.

IV. Módulo o longitud del radio = Raíz cuadrada de la suma del cuadrado del Zsum de X y del cuadrado del Zsum de Y.

V. Ratio = Zsum de la $\mathrm{Y}$ dividido por la longitud del radio.

VI. Ángulo $=$ Determinado según las siguientes indicaciones, siendo $\mathrm{f}=$ Arco seno de $\mathrm{Y} /$ Radio:

i. Si el vector se ubica en el cuadrante $\mathrm{I}(+,+)$ los ángulos corresponden a $0^{\circ}<\mathrm{f}<90^{\circ}$ luego el ángulo será $=\mathrm{f}$. La ubicación de la conducta condicionada en este cuadrante representa que la conducta focal y la condicionada se activan mutuamente.

ii. Si el vector se ubica en el cuadrante II $(-,+)$ los ángulos corresponden a $90^{\circ}<\mathrm{f}<180^{\circ}$, luego el ángulo será $=180^{\circ}-\mathrm{f}$. En este caso la conducta focal inhibe a la conducta condicionada, mientras que la conducta condicionada activa a la focal.

iii. Si el vector se ubica en el cuadrante III (-,-) los ángulos corresponden a $180^{\circ}<\mathrm{f}<270^{\circ}$, luego el ángulo será $=180^{\circ}-\mathrm{f}$, en este caso la conducta focal y la conducta se inhiben mutuamente.

iv. Si el vector se ubica en el cuadrante IV (+,-) los ángulos corresponden a $270^{\circ}<\mathrm{f}<360^{\circ}$, el ángulo será $=360^{\circ}-\mathrm{f}$, en este caso la conducta focal activa a la condicionada, mientras que ésta in- 
hibe a la focal (Castellano y Hernández-Mendo, 2003; Gorospe y Anguera, 2000; HernándezMendo y Anguera, 1999).

v. Pese a que en la representación vectorial del mapa de coordenadas polares aparecen todas las relaciones, únicamente se consideran significativas aquellas que el módulo del vector es superior a 1,96. Asimismo, el ángulo del vector, dependiendo del cuadrante en el que se encuentre, establecerá la naturaleza de la relación (Castellano y Hernández-Mendo, 2003; Hernández-Mendo y Anguera, 1999).

2. Representación gráfica de los vectores que indican el mapa conductual de cada una de las categorías condicionadas con respecto a la categoría focal.

\section{Resultados}

Se realizó el análisis secuencial usando las categorías focales con las diferentes categorías condicionadas y posteriormente su correspondiente análisis de coordenadas polares. A continuación se muestran los mapas conductuales estimados a partir de las categorías focales. Los resultados mostraron que para la categoría focal EF1 (Figura 3) aparecen en el cuadrante I, las conductas condicionadas CAN, CIR e IZQ, en el cuadrante III, la conducta LIN y, en el cuadrante IV, la conducta TRA. También se puede observar los resultados del análisis de coor- denadas polares para la categoría focal EF1 en la Tabla 2.

Figura 3. Representación del mapa conductual para la categoría EF1 como conducta focal (se representa el mapa conductual dividido en cuatro cuadrantes, con cada una de las categorías condicionadas como vectores en el eje X/Y y sus correspondientes coordenadas Zsum prospectivo $[\mathrm{X}]$ y Zsum retrospectivo [Y]).

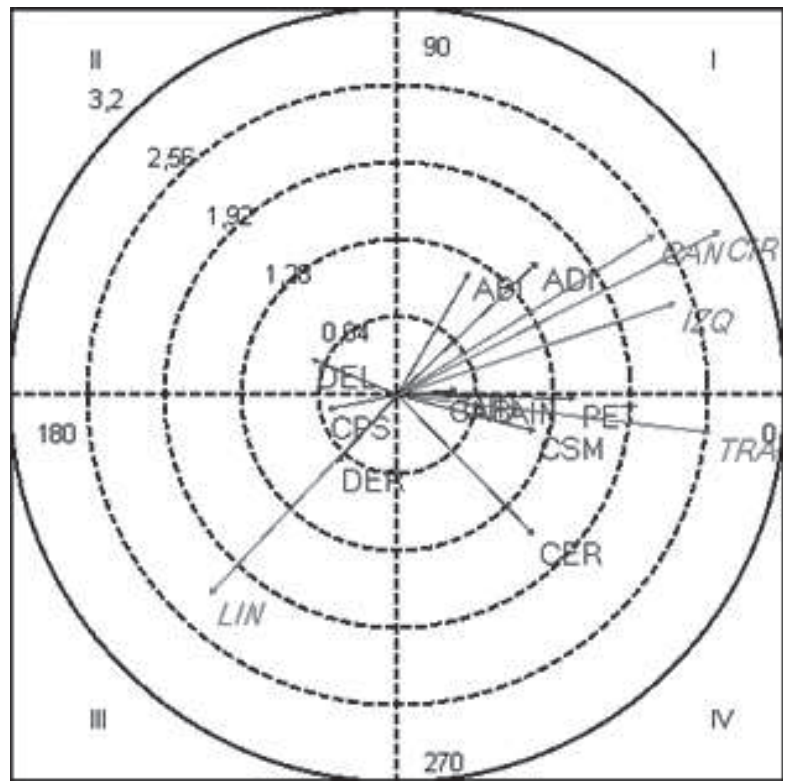

Tabla 2. Resultados del análisis de Coordenadas Polares para la categoría focal EF1.

\begin{tabular}{ccccccc}
\hline Criterio-Categoría & Cuadrante & P.Prosp. & P.Retrosp. & Ratio & Radio & Ángulo \\
\hline Acción táctica-ADI & I & 1,16 & 1,10 & 0,69 & 1,59 & 43,46 \\
Acción táctica-AIN & IV & 0,88 & 0,00 & 0,00 & 0,88 & 359,97 \\
Acción táctica-CAN & I & 2,12 & 1,32 & 0,53 & $2,50^{*}$ & 32,03 \\
Acción táctica-CSM & IV & 1,14 & $-0,30$ & $-0,26$ & 1,18 & 345,19 \\
Acción táctica-CPS & III & $-0,58$ & $-0,12$ & $-0,19$ & 0,59 & 191,22 \\
Tipo de técnica-LIN & III & $-1,55$ & $-1,65$ & $-0,73$ & $2,27^{*}$ & 226,82 \\
Tipo de técnica-CIR & I & 2,66 & 1,35 & 0,45 & $2,99^{*}$ & 26,91 \\
Tipo de técnica-GIR & I & 0,50 & 0,04 & 0,08 & 0,51 & 4,81 \\
Zona de golpeo-PET & IV & 1,47 & $-0,03$ & $-0,02$ & 1,47 & 358,67 \\
Zona de golpeo-CAB & I & 0,40 & 0,01 & 0,03 & 0,40 & 1,66 \\
Lateralidad-DER & III & $-0,50$ & $-0,56$ & $-0,74$ & 0,75 & 228,01 \\
Lateralidad-IZQ & I & 2,29 & 0,76 & 0,32 & $2,41^{*}$ & 18,36 \\
Pierna de golpeo-DEL & II & $-0,71$ & 0,30 & 0,39 & 0,78 & 156,85 \\
Pierna de golpeo-TRA & IV & 2,58 & $-0,31$ & $-0,12$ & $2,60^{*}$ & 353,22 \\
Guardia-ABI & I & 0,60 & 1,02 & 0,86 & 1,18 & 59,47 \\
Guardia-CER & IV & 1,13 & $-1,16$ & $-0,72$ & 1,62 & 314,17 \\
\hline
\end{tabular}

(*) Marca los vectores significativos (Radio>1,96). También se presentan las transiciones de las categorías y valores Zsum prospectivos y retrospectivos, cuadrante del vector, longitud del radio, ratio y ángulo del vector. 
Los resultados obtenidos para la categoría focal EF2 muestran, en el cuadrante I, las categorías condicionadas CAN y GIR, en el cuadrante II, las conductas condicionadas CAB y $\mathrm{ABI}$, en el cuadrante III, la conducta condicionada CIR y en el cuadrante IV, la conducta condicionada CER (Figura 4). Se pueden observar los resultados del análisis de coordenadas polares para la categoría focal EF2 en la Tabla 3.
Figura 4. Representación del mapa conductual para la categoría EF2 como conducta focal (se representa el mapa conductual dividido en cuatro cuadrantes, con cada una de las categorías condicionadas como vectores en el eje X/Y y sus correspondientes coordenadas Zsum prospectivo [X] y Zsum retrospectivo [Y]).

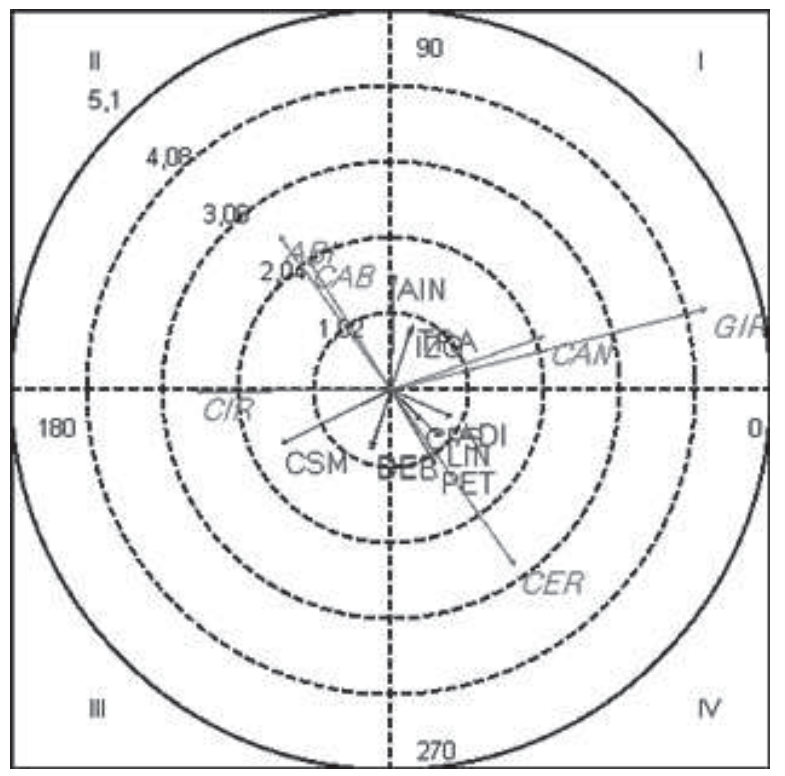

Tabla 3. Resultados del análisis de Coordenadas Polares para la categoría focal EF2.

\begin{tabular}{ccccccc}
\hline Criterio-Categoría & Cuadrante & P.Prosp. & P.Retrosp. & Ratio & Radio & Ángulo \\
Acción táctica-ADI & IV & 0,84 & $-0,37$ & $-0,40$ & 0,92 & 336,47 \\
Acción táctica-AIN & I & 0,04 & 1,59 & 1,00 & 1,59 & 88,52 \\
Acción táctica-CAN & I & 2,09 & 0,73 & 0,33 & $2,21^{*}$ & 19,34 \\
Acción táctica-CSM & III & $-1,50$ & $-0,74$ & $-0,44$ & 1,67 & 206,15 \\
Acción táctica-CPS & IV & 0,42 & $-0,42$ & $-0,71$ & 0,60 & 314,91 \\
Tipo de técnica-LIN & IV & 0,69 & $-0,65$ & $-0,69$ & 0,95 & 316,62 \\
Tipo de técnica-CIR & III & $-2,64$ & $-0,02$ & $-0,01$ & $2,64^{*}$ & 180,43 \\
Tipo de técnica-GIR & I & 4,29 & 1,10 & 0,25 & $4,43^{*}$ & 14,41 \\
Zona de golpeo-PET & IV & 0,62 & $-1,03$ & $-0,86$ & 1,20 & 300,92 \\
Zona de golpeo-CAB & II & $-1,13$ & 1,78 & 0,84 & $2,10^{*}$ & 122,35 \\
Lateralidad-DER & III & $-0,27$ & $-0,79$ & $-0,95$ & 0,84 & 251,30 \\
Lateralidad-IZQ & I & 0,27 & 0,83 & 0,95 & 0,88 & 71,83 \\
Pierna de golpeo-DEL & III & $-0,27$ & $-0,83$ & $-0,95$ & 0,87 & 251,70 \\
Pierna de golpeo-TRA & I & 0,30 & 0,89 & 0,95 & 0,94 & 71,72 \\
Guardia-ABI & II & $-1,52$ & 2,10 & 0,81 & $2,59^{*}$ & 125,89 \\
Guardia-CER & IV & 1,68 & $-2,38$ & $-0,82$ & $2,91^{*}$ & 305,29 \\
\hline
\end{tabular}

$\left({ }^{*}\right)$ Marca los vectores significativos (Radio>1,96). También se presentan las transiciones de las categorías y valores Zsum prospectivos y retrospectivos, cuadrante del vector, longitud del radio, ratio y ángulo del vector.

Los resultados obtenidos para la categoría focal EF3 únicamente muestran, en el cuadrante I, la categoría condicionada CIR (Figura 5). También se puede observar los resultados del análisis de coordenadas polares para la categoría focal EF3 en la Tabla 4. 
Figura 5. Representación del mapa conductual para la categoría EF3 como conducta focal (se representa el mapa conductual dividido en cuatro cuadrantes, con cada una de las categorías condicionadas como vectores en el eje X/Y y sus correspondientes coordenadas Zsum prospectivo $[\mathrm{X}]$ y Zsum retrospectivo $[\mathrm{Y}])$.

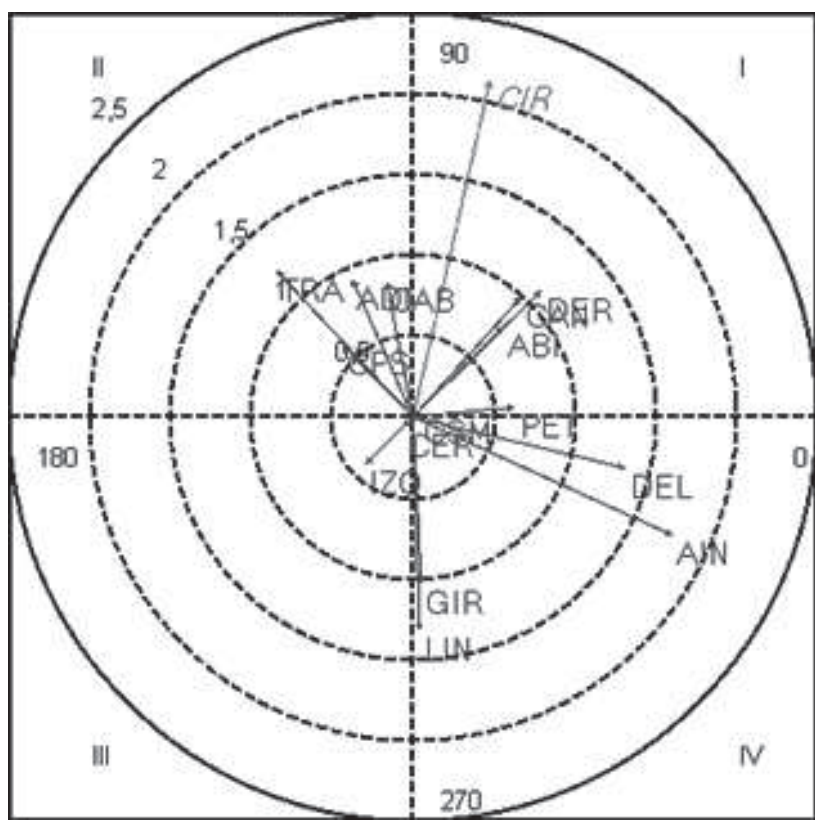

Tabla 4. Resultados del análisis de Coordenadas Polares para la categoría focal EF3.

\begin{tabular}{|c|c|c|c|c|c|c|}
\hline Criterio-Categoría & Cuadrante & P.Prosp. & P.Retrosp. & Ratio & Radio & Ángulo \\
\hline Acción táctica-ADI & II & $-0,37$ & 0,84 & 0,92 & 0,92 & 113,54 \\
\hline Acción táctica-AIN & IV & 1,58 & $-0,72$ & $-0,41$ & 1,74 & 335,54 \\
\hline Acción táctica-CAN & I & 0,66 & 0,73 & 0,74 & 0,98 & 48,06 \\
\hline Acción táctica-CSM & I & 0,04 & 0,03 & 0,60 & 0,05 & 36,77 \\
\hline Acción táctica-CPS & II & $-0,43$ & 0,44 & 0,71 & 0,62 & 134,88 \\
\hline Tipo de técnica-LIN & IV & 0,04 & $-1,30$ & $-1,00$ & 1,30 & 271,68 \\
\hline Tipo de técnica-CIR & I & 0,47 & 2,05 & 0,98 & $2,11^{*}$ & 77,17 \\
\hline Tipo de técnica-GIR & IV & 0,05 & $-1,01$ & $-1,00$ & 1,01 & 273,09 \\
\hline Zona de golpeo-PET & I & 0,62 & 0,06 & 0,10 & 0,62 & 5,53 \\
\hline Zona de golpeo-CAB & II & $-0,16$ & 0,82 & 0,98 & 0,83 & 101,11 \\
\hline Lateralidad-DER & I & 0,78 & 0,77 & 0,71 & 1,09 & 44,87 \\
\hline Lateralidad-IZQ & III & $-0,29$ & $-0,29$ & $-0,71$ & 0,41 & 225,31 \\
\hline Pierna de golpeo-DEL & IV & 1,29 & $-0,31$ & $-0,23$ & 1,33 & 346,52 \\
\hline Pierna de golpeo-TRA & II & $-0,83$ & 0,89 & 0,73 & 1,22 & 132,98 \\
\hline Guardia-ABI & I & 0,55 & 0,55 & 0,70 & 0,78 & 44,60 \\
\hline Guardia-CER & III & $-0,06$ & $-0,06$ & $-0,73$ & 0,08 & 226,97 \\
\hline
\end{tabular}




\section{Discusión}

El objetivo del presente estudio es establecer las diferentes relaciones entre las diversas categorías relacionadas con el comportamiento técnico-táctico de los taekwondistas y la consecución de punto/s en combate. Para conseguir el objetivo deseado, se realizó un análisis de coordenadas polares, se tomaron como conductas focales aquellas relativas a la efectividad (EF1, EF2 y EF3) y como conductas condicionadas aquellas relativas a las acciones técnico-tácticas (ADI, AIN, CAN, CSM, CPS, LIN, CIR, GIR, PET, CAB, DER, IZQ, DEL, TRA, ABI y CER).

En relación a la conducta focal EF1 (consecución de un punto), los resultados muestran que, en el cuadrante I (activa en perspectiva retrospectiva y prospectiva), dicha conducta focal activa las conductas condicionadas CAN, CIR e IZQ. Esto parece indicar que la anticipación, la técnica circular y realizada con pierna izquierda derivará en punto tras (o previo a) la realización de cuatro o menos acciones. Por ello, y debido a esta relación se sugiere el entrenamiento de este tipo de acciones. En esta línea, diversas investigaciones (i.e., Falcó et al., 2014; González, 2011; Menescardi et al., 2012; Menescardi et al., 2015) apuntan a la necesidad de realizar acciones anticipadas que puedan sorprender al contrario. El intento de sorprender al contrario, con acciones poco usuales buscando la consecución de puntos, ha sido planteado en trabajos previos (Chiodo et al., 2011; Falcó et al., 2014; Menescardi et al., 2015), lo cual podría explicar la realización de acciones con pierna izquierda, normalmente, la pierna no dominante. Sin embargo, para la realización de este estudio no se ha tenido en cuenta la dominancia de la extremidad inferior de los participantes; por ello, futuros estudios deberían abarcar este parámetro.

La relación entre la consecución de un punto y la realización de una técnica circular coincide con los resultados obtenidos en estudios previos, donde muestran que las acciones circulares son las más utilizadas en competición (Kwok, 2012; Matsushigue et al., 2009; Menescardi et al., 2012). A su vez, la mayor presencia de acciones circulares podría explicar la presencia en el cuadrante III (inhibición en perspectiva retrospectiva y prospectiva) de la conducta de condicionada LIN. Es decir, las técnicas lineales no se dan anterior ni posteriormente a la consecución de punto. Esta inhibición de la realización de la técnica lineal podría deberse a que este tipo de técnicas conllevan una menor fuerza de impacto (Serina y Lieu, 1991), por lo que se realizarían en menor medida durante un combate (Menescardi et al., 2012), prefiriéndose para esta misión (puntuar) las acciones circulares.

Finalmente, y relacionada con la conducta focal EF1, encontramos en el cuadrante IV (inhibición perspectiva retrospectiva y activación prospectiva) la conducta TRA. Parece ser que tras la consecución de un punto, las acciones que ocurren se caracterizan por ser realizadas con la pierna trasera. Esto podría explicarse a través de la lógica interna del deporte: si se tiene en consideración que tras la consecución de un punto no se detiene el combate, es posible el encadenamiento de acciones, donde la pierna de golpeo trasera del atacante (en la primera acción) se convierte en la pierna delantera (en la segunda acción) y el siguiente golpeo se realiza con la pierna trasera, y así sucesivamente, orientando el cuerpo y preparándolo para el siguiente golpeo. Normalmente, al reducir la distancia de combate entre competidores, las acciones se realizan con pierna trasera, ya que una menor distancia dificulta la movilidad y posibilidades de ejecución de acciones con pierna delantera. Estos resultados van en la línea de investigaciones previas (González, 2011) que muestran que la guardia con pierna trasera es la más utilizada y más efectiva, ya que es un golpeo contundente (Kong, Luky Hong, 2000) y con altas posibilidades de puntuar, así como de desequilibrar físicamente y emocionalmente al adversario (González, 2011).

En el mapa conductual estimado a partir de la categoría focal EF2 se puede destacar los siguientes aspectos relevantes: En el cuadrante I (activación en ambas perspectivas) aparecen las categorías condicionadas CAN y GIR. Ello también puede ser explicado a partir de la lógica interna del deporte, puesto que, reglamentariamente, únicamente se pueden conseguir dos puntos mediante una acción con giro. Asimismo, también parece ser que cuando un competidor realiza una acción con giro, el otro también la realiza (contraataque con giro), dándose en otros casos un intento por anticiparse a la acción de éste (ya sea con giro o con cualquier otra técnica). Esto podría explicar porqué ambas categorías aparecen en este primer cuadrante y explicaría a su vez, porqué la categoría condicionada CIR aparece en el cuadrante III (inhibición en ambas perspectivas). Asimismo, y al igual que ocurría con la conducta focal EF1, parecer ser que las acciones CAN están relacionadas no únicamente con la consecución de uno, sino también en la consecución de dos puntos, en ambas perspectivas. Sin embargo, investigaciones previas muestran que las anticipaciones son una de las acciones de contraataque que menos se utilizan (Falcó et al., 2014; González et al., 2011; Menescardi et al., 2015). Pese a esta menor utilización, diversos autores apuntan al gran potencial de las acciones anticipadas para sorprender al contrario y conseguir puntuar (Chiodo et al., 2012; Falcó et al., 2014; González, 2011). Por tanto, se sugiere el entrenamiento de estas acciones ya que parece ser que sorprenden al contrario y que, cuando se realizan, son efectivas.

Continuando con el mapa conductual estimado a partir de la categoría focal EF2, la aparición de las categorías condicionadas $\mathrm{CAB}$ y $\mathrm{ABI}$, en el cuadrante II (activación en perspectiva retrospectiva e inhibición en prospectiva), informan que tras la ejecución de una acción a la cabeza, generalmente manteniendo la guardia abierta, se tiende a realizar una ac- 
ción con giro, obteniendo dos puntos. Además, se observa que tras la consecución de puntos con una acción con giro, los deportistas tienden a inhibir la realización de acciones a la cabeza, bien por haber puntuado o por haber sido puntuados en contraataque, y también evitan adoptar la posición de guardia abierta. Estos resultados podrían atribuirse a que cuando un deportista estudia y/o enfrenta a un rival que tiende a realizar acciones a la cabeza adoptando la guardia abierta, la realización de acciones con giro parece inhibir su intención táctica de acciones a la cabeza desde guardia abierta. Así pues, el presente estudio permitiría a entrenadores y deportistas preparar de manera más adecuada y profunda los combates para adelantarse eficazmente a las acciones de los contrarios ya que no solo informa del tipo de acciones que tienden a realizarse o inhibirse, sino también de la adopción o no de la guardia desde la que se realizan.

En cuanto a las opciones de guardia adoptada, encontramos una relación en ABI y CER en los cuadrantes II y IV, respectivamente, para la categoría focal EF2 indicando que inhibe a la primera (ABI) en perspectiva prospectiva por estar en el cuadrante II y activa a la categoría condicionada CER por estar en el cuadrante IV. Parece ser que las acciones con giro (la única acción mediante la cual se consiguen dos puntos) se realizan con guardia abierta y que, esta misma situación (guardia abierta) favorece la realización de una acción con giro con pierna trasera (es decir, un giro de $180^{\circ}$ ) quedando situado el taekwondista en guardia cerrada tras la realización de la acción con giro. Estudios previos (González, 2011) muestran que los competidores están, normalmente, en una posición de guardia cerrada, preferiblemente con la pierna dominante en una posición atrasada para golpear con un mayor impacto, volviendo a adoptar la misma posición tras la realización de una acción con giro, por ejemplo, de $360^{\circ}$. Los deportistas de élite se caracterizan por la habilidad bilateral a la hora de realizar los golpeos (Falcó et al., 2009; Tang, Chang y Nien, 2007); por tanto, esta situación sugiere que los deportistas entrenen dicha habilidad bilateral ya que es posible que la necesiten durante la competición. Asimismo, se sugiere el entrenamiento de acciones con giro desde guardia abierta ya que parece ser la forma de conseguir dos puntos, así como el entrenamiento del posible bloqueo de dichas acciones por parte de sus oponentes.

En el mapa conductual estimado a partir de la categoría focal EF3 se observa únicamente una relación con la categoría condicionada CIR, en el cuadrante I. Ello implica que, independientemente de la acción previa o posterior a la consecución de tres puntos (ataque directo, indirecto, contraataque anticipado, simultáneo o posterior), ésta tiende a realizarse con una técnica circular. La mayor utilización de la técnica circular tanto para las conductas focales EF1 como EF3 permite seńalarla como relevante en combate ya que hay una clara presencia de dichas acciones antes y después a la conse- cución de 1 y 3 puntos. Este resultado parece coherente ya que únicamente se pueden conseguir 1 y 3 puntos mediante técnicas lineales o circulares, pero parece ser que la técnica lineal se utiliza en mayor medida debido a su mayor velocidad y fuerza de impacto (Serina y Lieu, 1991), por lo que también conviene entrenar dicho tipo de acciones.

Entre las limitaciones del estudio cabría señalar que no se ha tenido en cuenta el tiempo entre los diferentes retardos, lo cual podría suponer la existencia de relaciones entre acciones con un gran margen de tiempo entre ellas; sin embargo, según el reglamento no es posible que haya más de tres segundos de inactividad, siendo éste el periodo máximo que podría darse entre una acción y la posterior (WTF, 2012). Pese a las limitaciones comentadas, este estudio ofrece una base teórica y práctica sobre cómo aplicar la técnica de coordenadas polares en el taekwondo, así como ofrece unas orientaciones encaminadas al entrenamiento de los deportistas en función de los resultados obtenidos. Futuras líneas de investigación deberían centrarse en los patrones efectivos, desde una perspectiva retrospectiva que conlleven a la consecución de punto.

\section{Aplicaciones prácticas}

La técnica de análisis de coordenadas polares ha permitido comparar las relaciones que se establecen en la utilización de estrategias de interacción en el deporte de taekwondo. Poder representar en un mismo mapa conceptual las relaciones establecidas entre la categoría considera como focal y todas las demás categorías condicionadas permite dar cuenta de una manera clara de la evolución de dichas relaciones tanto en su significación (longitud del vector) como en la naturaleza de las mismas (cuadrante en el que se ajustan), lo que, sin duda, puede tener implicaciones técnico-tácticas para los entrenadores. Asimismo, se sugiere el entrenamiento de acciones anticipadas, lineales y con pierna trasera para la consecución de un punto ya que parecen ser que sorprenden al contrario y reportan una mayor eficacia en combate. Igualmente, se sugiere el entrenamiento de las acciones con giro de $180^{\circ}$ desde guardia abierta (finalizando en una guardia cerrada) ya que es la vía para conseguir dos puntos, conllevando también, el entrenamiento de bloqueos de dichas acciones, tanto durante la realización de las mismas (para evitar que los oponentes consigan sumar punto durante el tiempo en el que estén realizando la acción de giro) como por parte de sus oponentes para evitar la acción con giro.

Los resultados de la presente investigación han sido parcialmente financiados por los proyectos: Observación de la interacción en deporte y actividad física: Avances técnicos y metodológicos en registros automatizados cualitativos-cuantitativos, subvencionado por la Secretaría de Estado de Investigación, Desarrollo e Innovación del Ministerio de Economía y Com- 
petitividad [DEP2012-32124], durante el trienio 2012-2015; "Evaluación Psicosocial en Contextos Naturales: Deporte y Consumo (SEJ 444)", financiado por la Junta de Andalucía (Consejería de Innovación, Ciencia y Empresa); de los proyec- tos financiados por la Universidad Católica de Valencia "San Vicente Mártir” (UCV 2013-158-001), y de la Beca VALi+d de la Conselleria d'Educació, Cultura i Esport de la Generalitat Valenciana (ACIF/2014/268).

\section{Referencias}

1. Anguera, M.T. (1990). Metodología observacional. En J. Arnau, M.T. Anguera y J. Gómez Benito: Metodología de la investigación en Ciencias del Comportamiento (pp. 125-236). Murcia: Universidad de Murcia.

2. Anguera, M.T. (1997). From prospective patterns in behavior to joint analysis with a retrospective perspective. Colloque sur invitation "Méthodologie d'analyse des interactions sociales». Paris: Université de la Sorbonne.

3. Anguera, M.T. y Losada, J.L. (1999). Reducción de datos en marcos de conducta mediante la técnica de coordenadas polares. En M.T. Anguera (Ed.), Observación de la conducta interactiva en situaciones naturales: Aplicaciones. Barcelona: E.U.B.

4. Anguera, M.T., Blanco, A., Hernández-Mendo, A. y Losada, J.L. (2011). Diseños observacionales: ajuste y aplicación en psicología del deporte. Cuadernos de Psicología del Deporte, 11(2), 63-76.

5. Anguera, M.T., Espinosa, M.C. y Santoyo, C. (2002). Observación de la conducta interactiva en niños: Análisis de la intensidad interactiva diádica. Metodología de las Ciencias del Comportamiento, vol. especial, 34-36.

6. Anguera, M.T., Santoyo, C. y Espinosa, M.C. (2003). Evaluating links intensity in social networks in a school context through observational designs. En R. García Mira, J.M. Sabucedo Cameselle y J. Roma Martínez (Eds.), Culture, Environmental Action and Sustainability (pp. 286-298). Göttingen: Hogrefe y Huber.

7. Anguera, M.T. y Hernández-Mendo, A. (2013). La metodología observacional en el ámbito del deporte. E-balonmano.com: Revista de Ciencias del Deporte 9(3), 135-160. http:/www.e-balonmano.com/ojs/ index.php/revista/article/view/139.

8. Anguera, M.T. y Hernández-Mendo, A. (2014). Metodología observacional y psicología del deporte: Estado de la cuestión. Revista de Psicología del Deporte, 23(1), 103-109.

9. Banerjee, M., Capozzoli, M., McSweeney, L. y Sinha, D. (1999). Beyond kappa: A review of interrater agreement measures. Canadian Journal of Statistics, 27(1), 3-23.

10. Blanco-Villaseñor, A., Castellano, J. y Hernández-Mendo, A. (2000). Generalizabilidad de las observaciones de la acción del juego en el fútbol. Psicothema, 12(2), 81-86.

11. Blanco-Villaseńor, A., Castellano, J., Hernández-Mendo, A., SánchezLópez, C.R. y Usabiaga, O. (2014). Aplicación de la TG en el deporte para el estudio de la fiabilidad, validez y estimación de la muestra. Revista de Psicología del Deporte, 23(1), 131-137.

12. Camerino, O., Prieto, I., Lapresa, D., Gutiérrez-Santiago, A. y Hileno, R. (2014). Detección de T-patterns en la observación de deportes de combate. Revista de Psicología del Deporte, 23(1), 147-155.

13. Casolino, E., Lupo, C., Cortis, C., Chiodo, S., Minganti, C., Capranica, L. y Tessitore, A. (2012). Technical and tactical analysis of youth taekwondo performance. Journal of Strength and Conditioning Research, 26, 1489-1495.

14. Castellano, J. y Hernández-Mendo, A. (2003). El análisis de coordenadas polares para la estimación de relaciones en la interacción motriz en fútbol. Psicothema, 15(4), 569-574.

15. Castellano, J., Hernández-Mendo, A., Morales-Sánchez, V. y Anguera, M.T. (2007). Optimizing a probabilistic model of the development of play in soccer. Quality and Quantity, 41(1), 93-104.

16. Cochran W. G. (1954) Some methods for streghning the common $\chi^{2}$ test. Biometrics, 10, 417-451
17. Cohen, J. (1960). A coefficient of agreement for nominal scales. Educational and Psychological Measurement, 20, 37-46.

18. Chiodo, S., Tessitore, A., Lupo, C., Ammendolia, A., Cortis, C. y Capranica, L. (2012). Effects of official youth taekwondo competitions on jump and strength performance. European Journal of Sport Science, 12, 113-120.

19. Espinosa, M.C., Anguera, M.T. y Santoyo, C. (2004). Análisis jerárquico y secuencial de patrones sociales "rudimentarios" establecidos por niños pequeños. Metodología de las Ciencias del Comportamiento, Suplemento, 193-200.

20. Falcó, C., Álvarez, O., Estevan, I., Molina-García, J., Mugarra, F. y Iradi, A. (2009). Kinematic and kinematical analysis of Dominant and Non-Dominant kicking leg in a roundhouse kick in taekwondo. In A.J., Harrion, R., Anderson, y I., Kenny (Eds.). Scientific Proceedings of the 27th International Conference on Biomechanics in Sports (pp. 600603). Limerik: University of Limerik.

21. Falcó, C., Estevan, I., Álvarez, O., Morales-Sánchez, V. y HernándezMendo, A. (2014). Tactical analysis of the winners' and non-winners' performances in a Taekwondo University Championship. International Journal of Sports Science and Coaching, 9(6), 1407-1416.

22. Falcó, C., Landeo, R., Menescardi, C., Bermejo, J. L. y Estevan, I. (2012). Match analysis in a University Taekwondo Championship. Advances in Physical Education, 2, 28-31.

23. Fleiss, J.L. (1981). Statistical methods for rates and proportions. New York: Wiley.

24. González, C. (2011). Caracterización técnico-táctica de la competición de combate de alto nivel en Taekwondo. Efectividad de las acciones tácticas. Tesis doctoral no publicada. Barcelona: Universidad de Barcelona.

25. González, C., Iglesias, X., Mirallas, J. y Esparza, G. (2011). Sistematització de l'acció tàctica en el taekwondo d'alta competició. Apunts: Educació Física i Esports, 103(1), 56-57.

26. Gorospe, G. (1999). Observación y análisis en el tenis de individuales. Aportaciones del análisis secuencial y de las coordenadas polares. Tesis Doctoral. Vitoria: Universidad del País Vasco.

27. Gorospe, G. y Anguera, M.T. (2000). Modificación de la técnica clásica de coordenadas polares mediante un desarrollo distinto de la retrospectividad: aplicación al tenis. Psicothema, 12(2), 279-282.

28. Hermann, G., Sholz, M., Vieten, M. y Kohloeffel, M. (2008). Reaction and performance time of taekwondo top-athletes demonstrating the Baldung-Chagi. In Kwon, H., Shim, J., Shim, K., y Shim, S. (eds.). Proceedings of the 26th international Symposium on Biomechanics in Sports (pp. 416-419). Seoul, Korea.

29. Hernández-Mendo, A. y Anguera, M.T. (1999). Aportaciones de análisis de coordenadas polares a los deportes de equipo. En F. Guillén (Ed.), La Psicología del Deporte en España al final del milenio (pp. 169-175). Las Palmas: Universidad de Las Palmas de Gran Canaria.

30. Hernández-Mendo, A., Castellano, J., Camerino, O., Jonsson, G., Blanco-Villaseñor, A., Lopes, A, y Anguera, M. T. (2014). Programas informáticos de registro, control de calidad del dato, y análisis de datos. Revista de Psicología del Deporte, 23(1), 111-121.

31. Hernández-Mendo, A., López-López, J.A., Castellano, J., MoralesSánchez, V. y Pastrana, J.L. (2012). HOISAN 1.2: Programa informático para uso en metodología observacional. Cuadernos de Psicología del Deporte, 12(1), 55-78. 
32. Hernández-Mendo, A. y Anguera, M.T. (1998). Análisis de coordenadas polares en el estudio de las diferencias individuales de la acción de juego. En: Sánchez, M.P., López Quiroga Estévez, M.A. (eds.). Perspectivas actuales en la investigación de las diferencias individuales, (pp. 84-88). Madrid: Centro de Estudios Ramón Areces.

33. Herrero, M.L. (2000). Utilización de la técnica de coordenadas polares en el estudio de la interacción infantil en el marco escolar. Psicothema, 12(2), 292-297.

34. Iglesias, X., Gasset, A., González, C. y Anguera, M.T. (2010). Interacción competitiva y presión ambiental en deportes de combate: aplicación de la metodología observacional. Revista Iberoamericana de Psicología del Ejercicio y Deporte, 5, 267-282.

35. Kazemi, M, Casella, C. y Perri, G. (2009). 2004 Olympic Tae Kwon Do Athlete Profile. The Journal of the Canadian Chiropractic Association, 53, 144-152.

36. Kazemi, M, Waalen, J, Morgan, C y White A.R. (2006). A Profile of Olympic Taekwondo Competitors. Journal of Sports Science and Medicine, 5, 114-121.

37. Kim, J., Kwon, M., Yenuga, S. y Kwon, Y. (2010). The effects of target distance on pivot hip, trunk, pelvis, and kicking leg kinematics in Taekwondo roundhouse kicks. Sports Biomechanics, 9(2), 98-114.

38. Kong, P.W., Luk, T.C. y Hong, Y. (2000). Difference between Taekwondo roundhouse kick executed by the front and back leg. In Y. Hong, D. Johns, and R. Sanders (Eds.), Proceedings of 18th international symposium on biomechanics in sports (pp. 268-272). Hong Kong:University of Hong Kong.

39. Krippendorff, K. (1980). Content Analysis: An Introduction to its Methodology. Beverly Hills, CA: Sage.

40. Krippendorff, K. (2004). Content Analysis: An introduction to its methodology, (2nd ed.). Beverly Hills, CA: Sage.

41. Kwok, H. (2012). Discrepancies in fighting strategies between Taekwondo medalists and non-medalists. Journal of Human Sport and Exercise, 7, 806-814.

42. Landis, J.R. y Koch, G.G. (1977). The measurement of observer agreement for categorical data. Biometrics, 33, 159-174.

43. Matsushigue, K, Hartmann, K. y Franchini, E. (2009). Taekwondo: physiological responses and match analysis. Journal of Strength and Conditioning Research, 23, 1112-1117.

44. Menescardi, C, Bermejo, J.L., Herrero, C., Estevan, I., Landeo, R. y Falcó, C. (2012). Diferencias técnico-tácticas en taekwondistas uni- versitarios según sexo y categoría de competición. Revista de Artes Marciales Asiáticas, 7, 1-11.

45. Menescardi, C., López-López, J.A., Falcó, C., Hernández-Mendo, A. y Estevan, I. (2015). Tactical Aspects of a National University Taekwondo Championship in relation to Round and Match Outcome. Journal of Strength and Conditioning Research, 29(2), 466-471.

46. Perea, A. (2008). Análisis de las acciones colectivas en el fútbol de rendimiento. Tesis doctoral no publicada. Vitoria-Gasteiz: Universidad del País Vasco.

47. Perea, A., Castellano, J., Alday, S. y Hernández-Mendo, A. (2012). Analysis of behaviour in sports through Polar Coordinate Analysis with MATLAB. Quality and Quantity, 46(4), 1249-1260.

48. Sackett, G.P. (1980). Lag Sequential Analysis as a data Reduction Technique in Social Interaction Research. In D.B. Sawin, R.C. Hawkins, L.O. Walker y J.H. Penticuff (Eds.), Exceptional infant. Phychosocial risks in infant-environment transactions (pp. 300-340). New York: Brunner/Mazel.

49. Santos, V., Franchini, E. y Lima-Silva, A. (2011). Relationship between attack and skipping in taekwondo contests. Journal of Strength and Conditioning Research, 25, 1743-1751.

50. Santoyo, C. y Anguera, M.T. (1993). Evaluación ambiental: Integración de estrategias flexibles en situaciones naturales. En M. Forns y M.T. Anguera (Eds.), Aportaciones recientes a la evaluación psicológica (pp. 121-135). Barcelona: P.P.U.

51. Serina, E.R. y Lieu, D.K. (1991). Thoracic injury potential of basic competition Taekwondo kicks. Journal Biomechanics, 24(10), 951-960.

52. Tang, W.T., Chang, J.S. y Nien, Y.H. (2007). The kinematics characteristics of preferred and non-preferred roundhouse kick in elite taekwondo athletes. Journal of Biomechanics, 40(s2), S780.

53. Tornello, F., Capranica, L., Chiodo, S., Minganti, C. y Tessitore, A. (2013). Time-motion analysis of youth Olympic Taekwondo combats. Journal of Strength and Conditioning Research, 27, 223-228.

54. Tornello, F., Capranica, L., Minganti, C., Chiodo, S., Condello, G. y Tessitore, A. (2014). Technical-Tactical Analysis of Youth Olympic Taekwondo Combat. Journal of Strength and Conditioning Research, 28(4), 1151-1157.

55. Usabiaga, O. (2005). Evaluación de la acción de juego de la pelota vasca: aplicación en mano parejas. Tesis doctoral: Universidad del País Vasco.

56. World Taekwondo Federation (2012). Competition rules. Extraído desde http://www.wtf.org/wtf_eng/site/rules/competition.html. 